\title{
NEW DIRECTIONS AND DEVELOPMENTS IN ROBOTICS AND SITE AUTOMATION IN THE U.S.A.
}

\author{
Miroslaw J. Skibniewski \\ President (2000-01), International Association for Automation and Robotics in \\ Construction http://www.iaarc.org \\ Editor, Construction Technologies and Engineering, "AUTOMATION in \\ CONSTRUCTION" http://www.elsevier.com/locate/autcon \\ Professor of Civil Engineering, Purdue University, West Lafayette, Indiana 47907- \\ 1294, USA. \\ http://bridge.ecn.purdue.edu/ mirek
}

\begin{abstract}
This paper discusses examples of the latest developments in robotics technologies in the United States that may be of interest to construction robot systems developers. Both theoretical, far reaching research such as collaborative robotics as well as hardware systems oriented R\&D is included in the paper. Examples of the latest industry initiatives and consortia activities are also described. The paper concludes with observations about the current lack of direct interest by U.S. construction industry in robotics implementation, and about the U.S. leadership in information technology revolution encompassing, among others, the construction industry itself.
\end{abstract}

Keywords: robotics, automation, information technology, construction

\section{Introduction}

The population of robots nearly doubled over the last decade in North America alone, and they are becoming increasingly important in applications ranging from quality control to space exploration, surgery to the service industries. The industry recently has seen the emergence of new types of devices, including tiny micro- and nano-robots and robots with multiple arms or legs.

Popular attitudes toward robots have changed over the years. The fear that robots would replace workers has disappeared. Instead of displacing large numbers of employees in manufacturing, robots have brought about a more highly trained work force better capable of running robots and computers. There are many more trained people in robotics now, but some new challenges for robotics researchers are better human-robot collaboration interfaces, robot mobility and navigation in unknown surroundings, and better robot intelligence for service industries such as construction and for public transportation."
The number of robots per 10,000 manufacturing employees skyrocketed from 1980 to 1996 . For example, it increased from 8.3 to 265 in Japan, 2 to 79 in Germany, 3 to 38 in the United States and zero to 98 in Singapore. In roughly the same time, the world robot population surged, going from about 35,000 in 1982 to 677,000 in 1996 and an estimated 950,000 this year. In the five years from 1992 to 1997, the robot population in North America increased 78 percent, from 46,000 to 82,000 .

Robotics applications in the construction industry have been researched, explored and prototyped for the last 20 years. It is now over 16 years ago that the First International Symposium on Robotics in Construction was held in Pittsburgh, and much has been accomplished in the robotics technology since then. The International Association for Automation and Robotics in Construction (IAARC), its national clusters and partner organizations have been promoting the application of robots and automation technology in this large and diverse industry. As a result, automation and telecommunication 
technologies of today are far different from those of the last two decades.

Innovative technologies emerging over the first decade of the $3^{\text {rd }}$ millennium promise to affect virtually all aspects of everyday society, including health care, communication, manufacturing, transportation and, of course, construction. Robotics in the construction industry are in the large part based on advances in robotics developed first for other industries, and this trend is expected to continue.

\section{Emerging Core Technologies}

Engineers and scientists have already envisioned superbly efficient cars, "smart" offices equipped with wireless sensors, nanotechnology and a new class of miniature devices that dramatically speed up medical and biological testing, and electronic gear that runs for days on a single charge.

Nanotechnology will lead to ultra-compact video and audio equipment with far more storage capacity. More compact and versatile robots will enter the work force in a number of industries. While it is impossible to predict exactly what will happen, some future trends appear to be obvious. Fuel-efficient cars will likely travel about 80 miles on one gallon of gasoline and use a "hybrid" drive system: an electric motor powered by batteries or fuel cells, supplemented with a traditional fuel engine only when needed for acceleration and high performance. However, the success of hybrids will depend largely on the perfection of a control system that switches automatically from one drive to the other. With two propulsive sources, the use of both will need to be optimized automatically to make it all happen smoothly. Hybrid fuel technology may have a profound impact on the future of mobile robotics much needed in automated construction of roads and in transportation of building materials.

The automotive leap also will depend on the refinement of fuel cells, which generate electricity through electrochemical reactions between hydrogen and oxygen. In the coming decades, new types of fuel cells will likely evolve. Some visionaries predict that, before the middle of the next century, automobile fuel cells will generate enough electricity to supply an average-sized building.

Another near-term innovation will be the proliferation of very small, wireless electronic sensors the size of computer chips that will improve safety and make life easier in the workplace and at home. In general, most objects that surround us will be considered 'smart.' Some sensors will monitor the air for carbon monoxide and smoke. Others, linked via radio signals instead of wires, will automatically adjust the lighting and temperature. Employees may wear electronic cards or badges that specify their whereabouts. The badges might be programmed with a wearer's atmospheric and other personal preferences, much like a system designed for Microsoft's Chairman Bill Gates' home in the state of Washington, USA. Such innovations will become commonplace in the $21^{\text {st }}$ century. There will be thousands of these embedded chips, in the doorknobs, light bulbs, tables, chairs, all networked together. Similar sensors and electronic chips will be imbedded in structures such as bridges, road surfaces, foundations, and building walls to continuously monitor and report their condition and performance.

Advances in electronics and computers will have a direct impact on business travel, as improvements to videoconferencing make the long-distance medium much more realistic, perhaps even three-dimensional. Wireless Internet connections also will be refined on a commercial basis, and new computer software and hardware will make cellular phone communications more seamless. There will be no need to plug a computer into a telephone jack anymore. It will be possible to drive under an overpass, or inside a tunnel, and not have one's cell phone fade out. And there will be no need to worry about airplane flutter every time an airplane flies overhead. 
Meanwhile, innovative electronics and software will increase the operating efficiencies of equipment such as laptop computers and cellular phones so that they run longer on a single charge. These devices will use knowledge of what is running on the system and then intelligently shut down the parts that are not needed. In doing so, power will be conserved and batteries will last longer.

The higher efficiency will make wireless communications more practical as well. Global Positioning Systems already are available that use satellites to pinpoint an exact location of physical objects such as people and equipment. New software advances are making satellite-imaging systems very user-friendly that they might soon be accessible to everyone from farmers to real estate developers and to construction contractors as well. The software will handle the most mathematically rigorous work so that the systems can be operated by people with minimal technical skills. At the same time, the satellites themselves are improving and will soon be providing more data that can be used in numerous applications, including land development and transportation planning.

In other research, scientists expect to be able to merge biotechnology with computer science, creating a new class of miniature sensors that will be imbedded in a human body to deliver a variety of information useful in determining biological performance of human organs during the conduct of work. This experimental concept is called MIBBS, for 'micro-scale integrated bio-separation and bio-sensing systems,' and is currently at a development stage at Purdue University.

These and other developments are likely to lead to better and smaller robots for a nearly unlimited range of applications, from medicine to household maintenance, and to construction related uses.

\section{Progress in Research Laboratories}

A number of R\&D projects are currently underway throughout the United States that are of interest to the construction industry community. Several example projects are described below.

\section{Progress in Hardware Research}

Scientists and engineers have raised the possibility that machines constructed at the molecular level (nanomachines) may be used to cure the human body of its various ills. Nanorobots are nanodevices that will be used for the purpose of maintaining and protecting the human body against pathogens. They will have a diameter of about 0.5 to 3 microns and will be constructed out of parts with dimensions in the range of 1 to 100 nanometers. The main element used will be carbon in the form of diamond/ fullerene nanocomposites because of the strength and chemical inertness of these forms. Many other light elements such as oxygen and nitrogen can be used for special purposes. To avoid being attacked by the host's immune system, the best choice for the exterior coating is a passive diamond coating. The smoother and more flawless the coating, the less the reaction from the body's immune system. Such devices have been designed in recent years but no working model has been built so far. Although it is now difficult to imagine any potential applications of nanorobots in the construction industry, microrobots have already been prototyped and tested in a variety of tasks in the maintenance of built infrastructure. Trenchless technology is now becoming a mature application of this concept in the industry, including a larger scale of semiautomated devices. New pipelines may be installed or pipelines may be rehabilitated without the need for cut-and-fill excavation. Trenchless technology minimizes the cost of pipeline construction by avoiding excavation, minimize pedestrian and vehicular disruptions, retain landscaping, and reduce contractor's liability insurance premiums.

\section{Legged Locomotion}

Purdue University Robot Vision Laboratory has developed a vision-based quadruped walking robot system. The goal of this project was to design a legged robotic system that can sense, control, and plan its own locomotion in response to the conditions in the environment. Over the years it has become increasingly evident that all of these capabilities must work 
together for a legged robot to accomplish empirically interesting tasks.

The progress made so far in the design of legged robots has been mostly in the areas of leg coordination, gait control, stability, incorporation of various types of sensors, etc. This progress has resulted in the demonstration of rudimentary robotic walking capabilities in various labs around the world. The more stable of these robots have multiple legs, four or more, and some can even climb stairs. But what is missing in most of these robots is some perception-based high-level control that would permit a robot to operate with a measure of autonomy. Equipping a robot with perceptionbased control is not merely a matter of adding to the robot yet another module; the high-level control must be tightly integrated with the lowlevel control needed for locomotion and stability.

\section{Mobile Robot Navigation}

In the past decade, the Purdue University Robot Vision Laboratory has developed two unique reasoning and control architectures for vision-guided navigation by indoor mobile robots. Both of these systems use model-based vision to help a robot determine its location with respect to the environment. Both of these systems are fully operational and demonstrations of these are given routinely in the Laboratory.

The first of these architectures, called FINALE, allows a robot to navigate at approximately 8 meters per minute in the presence of stationary and moving obstacles. FINALE requires simple geometrical models of interior space. These models can be created simply by knowing the height of the ceiling and the locations of the principal corners in a hallway. The system can function even when some of these features are missing from the model map.

The second architecture, called FUZZY-NAV, uses neural-networks to keep a robot centered in the middle of a hallway and to generate steering commands when the robot needs to turn for either course correction or for avoiding obstacles. FUZZY-NAV needs only topological models of interior space. While the models are easy to create for FINALE, they are even more easily created for FUZZY-NAV. FUZZY-NAV is an extension using fuzzy logic for supervisory control of an earlier system, called NEURO-NAV, that also came out of this Purdue University laboratory.

\section{Caisson Construction 3-D Modeling System}

One of the critical links to the success of robotics in construction applications is the human-machine interface. This project is conducted at the National Robotics Engineering Consortium at Carnegie-Mellon under the sponsorship of Kajima Corporation. The researchers are developing a 3-D sensor system and graphical display to assist caisson construction equipment operators in digging a 42-meters deep caisson. The results of the terrain mapping are to be displayed in graphical form to human operators. These human operators are responsible for teleoperating excavating machines that are inside of the caisson. The human operators will be able to use the terrain-mapped display of the caisson to locate potential problem areas within the caisson structure and to determine what areas are stopping the caisson from sinking into the earth.

\section{Automated Underground Mining}

Another project underway at the National Robotics Engineering Consortium involves a system prototype involving a variety of excavating and transport machines configured to perform an underground mining operation.

Coal is mined using an ensemble of various machines. A continuous mining machine uses a spinning cutter head with "teeth" to break coal away from the face. The coal is deposited in shuttle cars for haulage to a permanent conveyor system and out of the mine. A roofbolting machine drills holes in the roof and inserts bolts to stabilize the roof and minimize the risk of collapse. Sometimes the miner and the bolter are combined into a single machine. This project concentrates initially on automating the functions of a continuous mining machine and roof bolting units.

\section{$\underline{\text { New Tactile Sensors }}$}

Scientists and engineers at Harvard University are currently involved in a Tactile Sensing 
Project involving development of new sensors, and integration of sensed information with real-time control. One new sensing technology already developed can localize high-frequency vibrations on the fingertip. They are working to use this sensor to measure friction at a single touch. They have also developed a fabrication technique for tactile pressure array sensors that results in a flexible, easily mounted sensor with very high sensitivity. These sensors are used with the two-fingered direct drive hand in the laboratory to study the role of tactile information in dexterous manipulation. The second sensing technology developed is based on deformable tactile sensors. Using this technology, on can reconstruct the deformed shape of the sensor on-line. This sensor is currently being used in manipulation tasks and in the development of a tool for minimally invasive surgery. It is expected that similar sensors may be utilized in a variety of nondestructive structural testing in the future.

Wireless Communication with Heavy Mobile Equipment

A vision of a job site where bulldozers "see" by sharing laser vision and terrain maps in real time has been created. In such a vision, a technician several miles away from the site can diagnose an earth-moving machine's malfunction. This communication all takes place without wires, antennae or complicated computer hardware.

Communication protocols differ between countries. There is also a significant problem of communication packets locating highly mobile machines that may move from site to site. There are also unique propagation problems at sites, not just the uneven terrain but the special problems of terrain changing shape significantly in real time. Add to this the complexities of multi-path interference. There are also a number of miscellaneous issues to be considered, for example:

- How do you integrate the pieces into a single system as you do with your wired computers?

- How do you deal with robustness?

- How do you deal with security?
Finally, although construction industry is one of the largest in most developed or developing economies, it is not large enough for a solution to its unique requirements to emerge spontaneously.

Caterpillar Corporation of Peoria, Illinois and the Information Networking Institute at Carnegie-Mellon University are working together under the university's Wireless Research Program to develop a mobile wireless communication system for construction equipment. Such a system assumes no availability of public networks and it is to benefit the construction and mining industries by supporting their field communications. This type of a system must provide a high-speed communication between earth-moving machines and from machines to the outside world. It needs to support field service by providing remote access to machines and giving field technicians direct access to up-to-date service manuals and data. Additional requirements include allowing dealers to do remote diagnosis of a machine's condition and enabling Caterpillar to field new, high-speed applications.

Many corporate and government owners of construction equipment fleets have unique needs. The owners often require a single site system that is easy to install, operate and maintain. The dealers want a simple technician interface - a rugged laptop as easy to use as a cellular phone - one that only needs turning on. This demanding set of requirements raises several issues. First, the system has to handle a wide variety of communications from data and file transfers to real-time data, voice telephony, and, someday, video, radar and laser images. In addition, the system needs to handle emergency stops and deal with hostile terrain. The system also has to work with wireless technology anywhere in the world.

The researchers have begun to meet these challenges. Using an IP-based architecturebasically a sophisticated "Internet in the sky"-they dealt with the issue of moving vehicles. They developed new processes for simple but high-speed networks in open-pit mines and quarries. They created a patented 
concept of intelligent protocols that use knowledge of the terrain and vehicle position to enhance reliability and performance.

After demonstrating their first year's work in Pittsburgh and at Caterpillar's Proving Grounds in Peoria, the researchers continued their efforts. They analyzed computer simulations of Caterpillar machines working in mines and quarries, and developed new protocols.

In a subsequent demonstration in Pittsburgh, the Carnegie-Mellon campus became the location of a supposed dealership and the neighboring Oakland area was used as the dealer's territory. Technology Park, two miles from main campus, was used to simulate a remote major construction site. Since remote mines and major construction sites are the kinds of locations where the largest Caterpillar machines demand the most sophisticated applications, it was an ideal way to test wireless data services.

The demonstration showed successful ad hoc networking, significant protocol improvement using terrain knowledge and transparent use of multiple networks - all while the networks were stressed with heavy communications traffic.

When Caterpillar implements this system into a fleet of "communications ready" vehicles, the mining and construction industries will be thrust into a world of high-speed, mobile communication technology. In addition, the products are being explored for NASA to apply to multiple spacecraft, space stations and clusters of surface robots as well as for the U.S. Army to use in mobile battlefield equipment. Other companies and agencies involved in this project include Bell Atlantic Mobile, Beckwith Machinery Company, Cisco, DARPA, EFData Corporation, Intel, Lucent Technologies, NASA, Glenn Research Center, Trimble Electronics, and the National Science Foundation.

\section{Autonomous Rover Technologies}

The primary goal of this project being conducted at the Carnegie-Mellon Robotics Institute is to research and develop the enabling technologies for autonomous planetary robot perception, position estimation, navigation, and integrated exploratory science from a robot, and validate such technologies through aggressive and rigorous field experimentation.

The recently executed research objectives include:

- Navigation and science from panoramic imagery: Prior research in wide field imaging developed teleoperated remote viewing and demonstrated its merits for robots, but fell short of the scope and benefits possible for automation with wide imagery. The attractive opportunity generated by capturing lateral and longitudinal views from a rover simultaneously has not been exploited. The team researches techniques for autonomous visual deduced reckoning, landmark based navigation, and scientific characterizations using panoramic imagery.

- Advanced radar perception and safeguarding: Sonar, stereo, and laser have dominated robot perception research, but each has liabilities and downfalls for application in space. Radar holds the prospect for modeling, safeguarding, and navigation from a space robot with advantages of operating in and through dust, in vacuum and atmosphere. The team investigates the merits of ultra highfrequency radar to detect objects, map terrain features, and even profile shallow subsurface geology in substantial dust accumulation during long traverses.

- Science data classification from multiple sensors: No "perfect" sensor or classification methodology exists for robustly distinguishing interesting science observations, like evidence for life, geologic anomalies, fossils, and meteorites among other rocks. The team has been developing a principled framework within which output from a variety of sensors and multiple classification algorithms are used to confirm or deny the detection of a scientific object of interest.

- Advanced rover autonomy: Extensive research has gone into obstacle detection and avoidance methods for autonomous robots. However, these methods largely rely on knowledge of robot characteristics (such as sensor coverage and mobility). 
Providing a robot with health monitoring and error recovery capabilities will allow the robot to notice that its turning radius has increased and incorporate this into its planning allowing a mission to continue even though a malfunction has occurred.

The researchers have been developing a general health monitoring capability capable of detecting failures in the drive, steering, and sensor components of the vehicle. An error recovery capability is also under investigation, which will use the error diagnosis to modify obstacle detection and avoidance behavior.

\section{$\underline{\text { Cognitive Robot Colonies }}$}

The primary objective of this work conducted at Carnegie-Mellon's Field Robotics Center and co-sponsored by Defense Advanced Research Project Agency is to uncover the basic principles that will best govern a group of robots trying to do useful work in difficult and hazardous environments, including construction sites. The foundation of the research begins with the idea that robot existence must be modeled probabilistically. Robots, like humans, are subject to physical laws and can be damaged or destroyed by both random and intentional events. In the extreme environments posed by space exploration, military operations, fire-fighting, nuclear cleanup or dangerous construction, the likelihood that robots will be injured is amplified. In many situations, the danger posed is so great that a single robot expected to perform adequately in these scenarios must be designed to mitigate every conceivable circumstance. Clearly, this task is either very difficult or impossible for most operations.

A promising approach is to use tightly coordinated groups, or colonies, of smaller, simpler robots to perform tasks in these dangerous locations. The fundamental advantage of this approach is redundancy of systems and mechanisms. If managed properly, the loss of a robot, although painful, will not be catastrophic and task execution capabilities will degrade gracefully across multiple robot failures. While very promising, the implementation of these ideas into a working system is fraught with the difficulties inherent in any highly redundant system.
Specifically, the work at Carnegie-Mellon is focusing on the following major problem areas:

- Behavioral Strategies for Colonization: What is the "critical mass" of agents necessary to form an effective colony? Once formed, what kind of internal, environmental, and task-level cues can be monitored to maintain the optimal colony through pruning and growth behaviors? Are there times when it is more efficient to disband the colony and work at the level of the individual robot?

- Group Learning Algorithms: Can the sharing of experiences amplify the effectiveness and minimize the convergence time of the learning processes within a robot colony? What is the proper mix of individual and group learning strategies? Can effective learned behaviors be distributed as a commodity within the colony?

- Protected and Secure Resources: What is the best way to distribute data and processes within the colony to insure that loss of robots will minimize loss of learned knowledge and data? Once losses are realized, what is the best way to redistribute existing information to make it more secure? What are the most efficient and secure methods of routing critical information and data within the colony and to the outside world?

- Task-Level Reprogramming: Can we obtain an efficient system for doing real work in hazardous locations using behavioral seeds from individual robots to grow a task-specific organization? If so, what is the optimal matrix of basic behaviors and the learning algorithm required to foster this system?

- Scalability: Robot colonies that rise as a dynamic response to general task-level requests will need to have great variability in their computational, power consumption, communications, and physical capabilities. Thus, robot system architecture must scale to problems at both ends of the spectrum. Can we make a colonization architecture that supports ten 
robots in one task and ten thousand for another?

- Although the focus of this work is fundamental, the ultimate measure of success of any robotic system should be evaluated in terms of performing useful work in a practical job scenario. For this reason, one may test the validity of the proposed concepts with respect to the task of Distributed Mapping of Urban Environments.

The unique feature of the proposed distributed mapping system, and the eventual metric of its success, will be its ability to pursue this task when faced with multiple robot failures. The initial demonstration, tentatively scheduled for the fall of 2001, will be to deploy ten small robots into a "mock-up" of an urban facility. These robots will form a colony whose sole purpose is the generation of a map of this area. After an initial period during which basic distributed mapping operation is demonstrated, project sponsors will be asked to "disable" robots of their choice and observe the reaction of the colony to this loss. This process will continue until critical mass is lost and the colony is unable to function in terms of its primary mission. Thus, observers will be given an "on-line" demonstration of how our system adapts to multiple and catastrophic failures.

The list of major research tasks includes the following:

1. Formalize the core components of free market architecture.

- Implement architecture and evaluate performance in simulation.

- $\quad$ Extend architectural capability to accommodate exploration of partially known worlds.

- $\quad$ Extend architectural capability to accommodate robot death.

- Evaluate architectural performance on real test bed with four robots.

- $\quad$ Extend architectural capability to accommodate multiple roles.

\section{Map Reconstruction}

- Robust matching of features between images taken at arbitrary positions by different robots, and recovery of robot poses and scene geometry.

- $\quad$ Recovery of dense depth maps from images from robots at arbitrary positions.

- Generation of texture-mapped realistic environment model from the reconstruction for presentation to users.

- $\quad$ Integration of multiple maps into a combined representation of the environment.

- Use of the geometric representation for reasoning about occlusions and view planning.

\section{Communal Learning}

- Develop communal learning techniques in which the colony shares experiences (data gathering) and the computational burden of learning.

- Prove that concepts can be learned by the colony that are beyond the capabilities of the individual.

- Show the benefit of having learned information preserved across the colony.

- $\quad$ Also show that the colony can reuse and build upon previously learned skills and behaviors. In situations, where new generations of robots replenish those consumed, this will lead to a progressive society where new generations can advance beyond the previous generation.

- $\quad$ Develop a "buddy" system for insuring fatal state information survives. Communal learning can then learn fatal situations and actions. In many situations, robot sacrifice will be required. Techniques will be developed that will insure that the most information is gained for the colony from any sacrifice.

- $\quad$ Fit communal learning into the free market architecture. By making experiences, computation, and new or refined behaviors/information a commodity, communal learning will be part of distributed market architecture.

- $\quad$ Show that communal learning can lead to more accurate and 
meaningful negotiations. This will be done by learning transaction confidence and expected costs.

- Apply communal learning to: mobility models, behavior parameters, transaction models, maps and enemy tactic models for individuals and teams.

4. Robot Test Bed

- $30 \mathrm{~cm} / \mathrm{s}$ reliable obstacle navigation.

- Integration of Firewire hardware on each platform.

- Integration of audio hardware on each platform.

- Data Abstraction Module for image acquisition (both streaming and static forms) and audio clips.

- Multi-link routing module that enables point-to-point communication through multiple intermediaries to compensate for limited range in our wireless hardware.

- Augment sonar-based obstacle avoidance with image-based approach.

- Characterize odometric data (linear and angular drive vs time).

- Develop network resource manager that empowers robots to obtain information and physical services from others.

- Design and integrate mounting brackets for audio and imaging hardware.

Related future work may also include determination of the collective intelligence of a colony of robots in the performance of given work tasks, based on theoretical foundations presented in the forthcoming book by Szuba (see References).

\section{Development of Standards for Automated Construction}

Researchers at the National Institute for Standards and Technology are currently involved with three projects related to construction site automation:

- Non-Intrusive Scanning Technologies for Construction Status Assessment. This project is intended to initially benefit large earthmoving projects and aims at enabling the use of scanning technology by the industry for near real-time, inexpensive assessment and tracking of construction status. The challenges include a statistically based calibration of laser device and traceability to NIST measurement standards, development and evaluation of advanced computational models/ algorithms for generating 3-D surfaces through mathematical optimization, automatic spatial registration of multiple point clouds, development of a mobile platform and spatial registration of platform, and development of automated object classification and recognition methods.

- Real-time Construction Components Tracking. The objective is to provide infrastructure necessary for the industry to achieve real-time identification and position tracking of manufactured components on a construction site. The challenges include the development and integration of advanced measurement systems for high accuracy tracking of position and orientation for manufactured objects on a construction site, defining interoperability needs for field sensors and wireless communication systems for part tracking, and developing methodology for defining fiducial points for unique object position and orientation determination.

- Site Measurement System Interoperability and Communications Standards. The objective is to enable the use and integration of multi-functional, heterogeneous sensor arrays by the industry to achieve cycle time reductions in construction. The challenges include the development of data representation/ interoperability standards and communication protocols that industry will accept and implement, the design of standards/ protocols that work well over wireless networks, facilitation of sensors/ user mobility about sites, interoperability assessment and field testing of standards, and determination of accuracy and reliability of field sensors.

\section{State of Practice in Robotics for Construction- Related Applications}

As determined by researchers over the past two decades, practical use of robotics in challenging environments such as a construction site depends on the ability of robots to move around, sense the environment, process the data and information received and 
reason based on available information - all with sufficient levels of redundancy to insure reliable field performance. A number of current and recent projects conducted in American laboratories address various aspects of these prerequisites.

The All-Terrain Robot Line from I-Robots, Inc. features four-wheel drive, differential steering, pneumatic knobby tires, long running time and a wide range of optional equipment and accessories. The ATRV family of robots is capable of performing work tasks in heavy terrain. These robots are built around RWI's

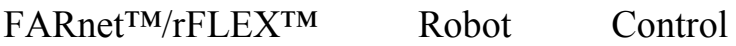
Architecture, and the versatile MOBILITYTM motion control package. The system includes a proprietary Robot Software Development Environment. Applications are developed once and may be run on any robot in the ATRV family without re-coding or porting. In addition, I-Robots, Inc. supplies wireless communications based on RS-232 standard, on-board computers, scanners, vision systems, joystick controls and navigation sensors for heavy terrain locomotion such as accelerometers and compass-tilt sensors, for all ATRV outdoor robots.

The NASA Space Telerobotics Program is an element of NASA's ongoing research program, under the responsibility of the Office of Space Science. The program is designed to develop telerobotic capabilities for remote mobility and manipulation, by merging robotics and teleoperations and creating new telerobotics technologies. Space robotics technology requirements can be characterized by the need for manual and automated control, nonrepetitive tasks, time delay between operator and manipulator, flexible manipulators with complex dynamics, novel locomotion, operations in the space environment, and the ability to recover from unplanned events. To meet these needs, the program is focused on the following goal: To develop, integrate and demonstrate the science and technology of remote manipulation such that by the year $2004,50 \%$ of the EVA-required operations on orbit and on planetary surfaces may be conducted telerobotically. The Space Telerobotics Program consists of a wide range of tasks from basic scientific research to applications developed to solve specific operations problems. The program is focused on three specific mission or application areas: on-orbit assembly and servicing, science payload tending, and planetary surface robotics. Within each of these areas, the program supports the development of robotic component technologies, development of complete robots, and implementation of complete robotic systems focussed on the specific mission needs. These three segments align with the application of space telerobotics to the class of missions identified by the potential space robotics user community. The objective of each of these program areas, from base technology development through systems applications, is to provide the technology for space telerobotics construction and related applications with sufficient validation that the designers of future spacecraft can apply the technology with confidence.

Turning to terrestrial disaster recovery sites, the Chernobyl nuclear power plant unit-4 in the Ukraine experienced a powerful explosion on April 26, 1986. Under extreme conditions, the Chernobyl Sarcophagus was constructed within six months of the explosion. This year, RedZone's Pioneer system will enter the damaged Chernobyl Nuclear Power Plant Unit4 reactor to sample and characterize environmental and structural conditions of the Unit-4 shelter, which was built six months after the April 26, 1986 explosion. Pioneer's goal is to provide data to aid in stabilization and remediation planning. Pioneer will be conducting structural assessments including:

- Visually surveying structural elements, walls, floors, and ceilings for damage, cracking, or other deterioration.

- Taking samples of concrete to determine the physical properties or degradation due to prolonged exposure to high radiation fields and other hostile environmental conditions.

Environmental assessment includes tasks such as:

- Measuring the gamma field intensity and neutron flux, and

- Locating and estimating the volumes of fuel containing material (FCM).

Pioneer includes mobility platform and sensor/tooling packages, such as a coreborer, 3D mapper, manipulators, and environmental sensors that measure temperature, humidity, and radiation levels. Pioneer will be equipped with shovel and arm. Two of Pioneer's 
sensor/tooling packages include a plow bucket for pushing and lifting gravel and other debris, as well as a fully electric manipulator that can pick-up and move a variety of objects.

Houdini produced by RedZone of Pittsburgh is a tethered, hydraulically-powered, track-driven work platform with a folding frame chassis that allows it to fit through confined access ways as small as 22.5 inches ( 0.57 meters) in diameter. Modeled after bulldozers and backhoes from the construction industry, and hardened to work in nuclear and other hazards, Houdini features a rugged, intuitive work platform, rugged design and sturdy construction that make it well-suited for the rigors of waste mobilization and other heavy work tasks. The robot features low-voltage servo-valving, spark-proof hydraulic operation, environmentally safe hydraulic fluid, and selfcollapsing capability (under gravity) for failsafe removal. Houdini can travel on, over, and through materials ranging in consistency from concrete to sludge to liquid. The robot system is fully submersible, allowing material handling to be performed on tank contents. Houdini also provides simple material handling similar to conventional earth-moving equipment, such as bulldozers and backhoes, in conjunction with a variety of dislodging, retrieval, and conveyance technologies. Originally designed to support the retrieval of nuclear waste from the U.S. Department of Energy storage tanks, Houdini has already been successfully used for that purpose in numerous tanks at the Oak Ridge National Laboratories. Houdini was developed by RedZone in cooperation with Federal Energy Technology Center and Fernald Laboratory.

Another product of RedZone Robotics, Inc., Rosie, is a heavy-payload, long-reach remote work vehicle, used in decontamination and decommissioning applications. A rugged mobile platform with advanced tooling and controls, Rosie combines the ability to dismantle an entire structure with the dexterity to decontaminate selected building components. Rosie deploys a wide range of tools and sensors from her heavy manipulator or locomotor deck. Variable-speed motion control allows an operator to position tools quickly to perform desired work tasks. In addition, Rosie's work envelope enables floorto-ceiling reach. A single operator stationed at a control console controls the robot. Primary system functions, such as boom, locomotor, system power, tether, and cameras, are operated using switches and joysticks on the desktop. Less frequently used functions and status information are accessed through a touch screen. Three video monitors with quadsplitting capability provide views of all onboard cameras. To maximize productivity, the operator can adjust the display location of any camera view. Additional video monitors can also be provided. Modular design of the robot allows expedient maintenance and deployment of alternate tools, such as a mechanical scabbler, sealant spray, reciprocating saw, plasma torch, pulverizer, wet/dry vacuum, and dexterous manipulator(s). At Argonne National Laboratory, Rosie works to dismantle the CP-5 experimental reactor. Though radiation levels at the work site have been higher than anticipated, this robot continues to be a reliable and flexible tool; maintaining a heavy and consistent workload, while preventing radiation exposure to human workers.

\section{Academic, Governmental and Industrial Initiatives}

The construction industry faces special challenges in reaping the full benefits of the automation and information technology revolution that has brought and continues to bring rich rewards to many industries. These include low R\&D investment, fragmentation, and its unique project-oriented character. Purdue University and the Construction Industry Institute developed a web site featuring the latest developments in advanced construction technologies <http://www.newtechnologies.org/ECT/>. The site serves as a vehicle for information transfer from research laboratories and technology developers in industry into the hands of actual and potential end users. A number of other web sites exist which provide relevant links to information sources related to professional and trade organizations in the construction industry, and to web-based project management services and other information technology resources.

The American Society of Civil Engineers established the Civil Engineering Research Foundation, which along with its affiliated "Innovation Centers" sponsors and organizes 
annual conferences and conducts an annual award scheme aimed at promoting the most promising innovations in design and construction. Additionally, ASCE operates a Field Sensing and Robotics Committee which organizes biennial conferences devoted to "Robotics for Challenging Environments." An organization based at the University of Michigan sponsors an annual construction innovation NOVA award based on practical impact of the innovation on construction industry practice.

The FIATECH (Fully Integrated and Automated Project Processes) Consortium has been established as a Construction Industry Institute affiliated, collaborative, not-for-profit research consortium conducting leveraged research and development of Fully Integrated and Automated Project Processes. Achieving this objective requires a breakthrough change in technology intensive processes. The first task involves a seamless integration and management of project information within the context if an entire facility life cycle and enterprise-wide resource planning system. The second tasks will bring about wireless data from the construction site into the project management information loop. FIATECH will conduct leveraged $R \& D$ in partnership with suppliers, with firms in the software/information technology industries, and with the public sector. Likely public sector partners include the National Institute of Standards and Technology, The U.S. Department of Transportation, The Army Corps of Engineers, and the General Services Administration.

\section{Conclusion}

The above overview of automation related activities in the United States indicates high volume of robotics research and development on behalf of industries outside of the construction sector, but with similar work characteristics as those typical on construction sites. Very little robotics R\&D activity takes place aiming directly at on-site applications. Historically, most robotics R\&D aimed at tasks related to maintenance and repair of built facilities.
However, there is certainly an information technology revolution taking place in industry, particularly in the area of data acquisition methods, metrology and internet based multimedia communications, with direct impact on construction procurement, project planning, design and construction efforts (see References). It is in this aspect of construction automation that the United States is leading the way, and it is likely to retain its leadership in the foreseeable future.

\section{REFERENCES}

Abduh, M.: "Utility Assessment of Electronic Networking Technologies for Design-Build Projects," unpublished Ph.D. dissertation, School of Civil Engineering, Purdue University, West Lafayette, Indiana, July 2000

Cheok, G., Lipman, R., Witzgall, Ch., Bernal, J., Stone, W.: "NIST Construction Automation Program Report No. 4: Non-Intrusive Scanning Technology for Construction Status Determination," NISTIR 6457, Building and Fire Research Laboratory, National Institute of Standards and Technology, Gaithersburg, Maryland, January 2000

Ciesielski, C., ed.: “ Proceedings, International Conference on Automatic Data Collection Technologies in Construction, Las Vegas, Nevada, March 28-30, 2000

Field Robotics Center, Carnegie-Mellon University, Cognitive Colonies Project, http://www.frc.ri.cmu.edu/ sthayer/sdr/ (July $30,2000)$

Johnson, D. and Bennington, B.: "Mobile Internet Protocol Performance and Enhancements Over ACTS", Proceedings, Advanced Communication Technology Satellite (ACTS) Conference 2000, Sixth KaBand Utilization Conference, NASA Glenn Research Center, Cleveland, Ohio, May 31, 2000 .

Leger, P.: "Automated Synthesis and Optimization of Robot Configurations: An Evolutionary Approach," doctoral dissertation, Robotics Institute, Carnegie- Mellon University, December 1999. 
Nof, S., ed.: "Handbook of Industrial

Robotics," $2^{\text {nd }}$ Edition, John Wiley \& Sons,

New York, 1999

Szuba T.: "Computational Collective Intelligence," Wiley Series on Parallel and Distributed Computing (forthcoming)

Wetzel, J., Bernold, L., Stone, W., eds.: Proceedings, Robotics 2000 - The 4th International Conference, Exposition, and Demonstration on Robotics for Challenging Situations and Environments, Albuquerque, New Mexico, American Society of Civil Engineers, February 28 - March 2, 2000 